\title{
Free vibration analysis and optimal design of adhesively bonded double-strap joints by using artificial neural networks
}

\author{
Qi Guo a* (1), Suian Wang ${ }^{\mathrm{a}}$ \\ a School of Astronautics, Northwestern Polytechnical University, Xi’an, China. Email: npuguoqi@qq.com, hzwangsuian@qq.com \\ * Corresponding author
}

http://dx.doi.org/10.1590/1679-78255878

\begin{abstract}
This study investigates the free vibration characteristics of an adhesively bonded double-strap joint with viscoelastic adhesive layer. To simplify the spatial finite element mesh generation and efficiently model the adhesively bonded joint, a layerwise plate finite element was extended to accommodate to the modeling of the joint, where the joint structure is treated as a special sandwich laminate. The proposed method was validated by three-dimensional finite element analysis and then applied to generate sampling points for training artificial neural networks (ANNs). The effects of the adhesive material properties and joint geometrical parameters on the joint dynamic characteristics were investigated in detail using the trained ANNs. The optimum design problem is defined as a multi-objective optimization problem considering maximizing the first natural frequency and corresponding loss factor while minimizing the total structural weight. The nondominated sorting genetic algorithm combined with the ANNs were employed to tackle the problem. The proposed method provides a computationally efficient alternative for analyzing and optimizing the adhesive double-strap joints.
\end{abstract}

\section{Keywords}

layerwise finite element, double-strap joint, free vibration, optimization, artificial neural network

\section{Graphical Abstract}

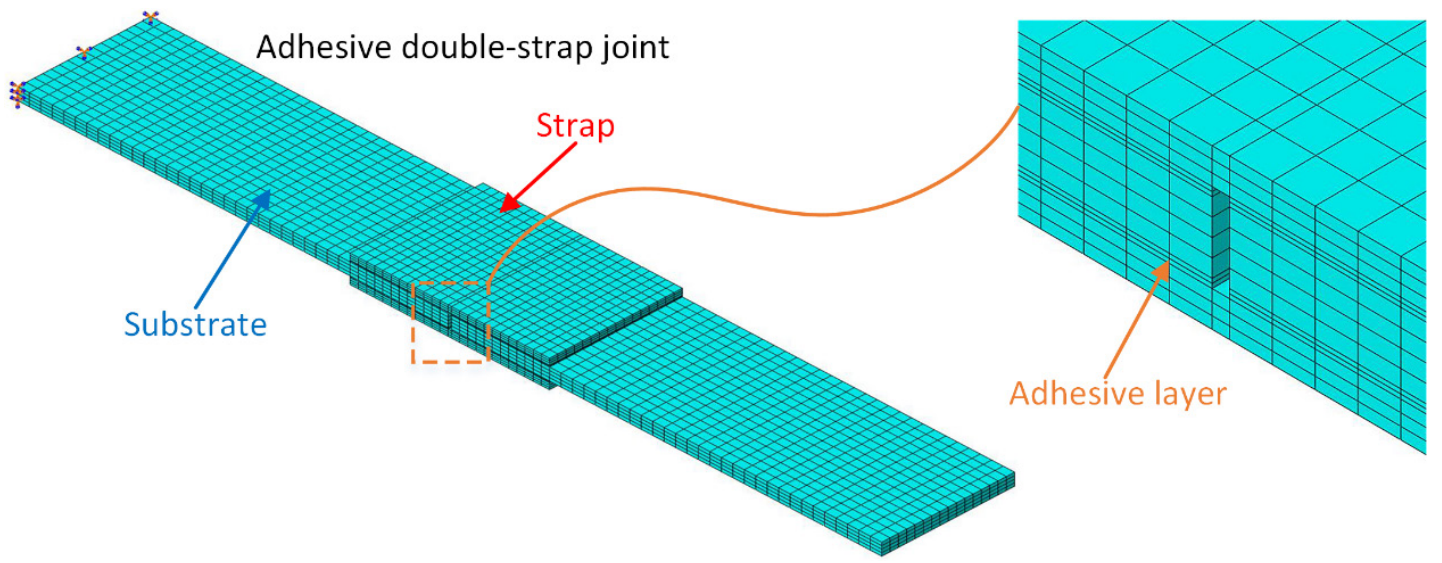




\section{INTRODUCTION}

Adhesively bonded joints are widely used in various industrial fields consisting of manufacture, aerospace, marine, automotive and civil structures. Compared to traditional joints such as bolting, riveting and welding, adhesive joints present many advantages in terms of reducing the overall weight, spreading stresses uniformly and bonding both similar and dissimilar materials. Several factors including design, materials, manufacturing methods and the accuracy of strength prediction influence the reliability of the adhesive joints. In order to design the adhesively bonded structures efficiently, it is necessary to understand their static and dynamic characteristics. The vibration characteristics of adhesively bonded joints are important, since harmonic loads or short period impact loads may weaken the joint strength, introducing fatigue damage and affecting the dynamic properties of such structures. Consequently, the investigation for the joint vibration properties have been performed by many researchers using analytical equations, experimental tests and finite element analysis.

Initially, analytical models were developed to model and analyze the vibration behaviors of the adhesively bonded joints. Saito and Tani (1984) developed an analytical model for predicting the natural frequencies and damping of the longitudinal and transverse vibration of a single-lap joint. To investigate the transverse vibration of a bonded beam with lap joints, analytical and experimental studies were carried out by Rao and Crocker (1990). He and Rao (1992a, 1992b) and Rao and $\mathrm{He}$ (1992a, 1992b) derived the governing equations of motion using energy method and Hamilton's principle to investigate the longitudinal and transverse dynamic responses of single-lap and double-strap joints bonded by viscoelastic adhesive. Rao and Zhou (1994) presented a mathematical model to study the vibration and damping of a bonded tubular lap joint under fixed-fixed boundary condition. Vaziri et al. (2001) and Vaziri and Nayeb-Hashemi (2002) dealt with the effects of voids on the joint dynamic responses by theoretical investigation. Ingole and Chatterjee (2011) studied the coupled longitudinal and transverse vibration of a single-lap adhesive joint with free-free configuration theoretically and experimentally. Based on an improved shear-lag model, a new analytical solution was proposed by Almitani and Othman (2016) to capture harmonic responses of adhesive joints. Comparing the results predicted by the solution to finite element solutions, good agreement was obtained. Challita (2018) established a mathematical model to research the dynamic behaviors of a bonded lap joint with a void implanting in the overlap. Though analytical solutions have the advantages of giving closed-form solution and are able to carry out preliminary analysis, important assumptions and simplifications would be required. Moreover, the joint three-dimensional nature together with its increasing complex geometry increases the difficulty of obtaining the governing equations of overall system. Therefore, it is infeasible to only adopt the analytical equations to study the joint vibration characteristics.

To overcome the drawbacks presented by analytical solutions, finite element methods (FEM) are adopted. Ko et al. (1995) and Lin and Ko (1997) employed a combination of adhesive interface element and plate element to analyze the dynamic responses of laminated plate bonded by adhesive. He and Oyadiji (2001) applied FEM to investigate the effects of adhesive material properties on the transverse free vibration characteristics of single-lap cantilevered beams. They found that the adhesive Young's modulus has significant influence on the transverse natural frequencies, whereas the Poisson's ratio has minor effects. Similarly, in a study (Xing et al., 2011), the effects of adhesive geometrical parameters on the transverse free vibration properties of the same joint were explored. By means of FEM, a research was conducted by Kaya et al. (2004) to investigate the effects of adhesive thickness and structural damping on the mode shapes, natural frequencies and transfer receptances of a single-lap joint, and the structural damping was found to decrease the resonance amplitudes greatly. He $(2012,2014)$ predicted the dynamic responses of a single-lap joint by FEM and validated the prediction through experimental tests. Gunes et al. $(2007,2010)$ implemented FEM, ANNs and genetic algorithm for the free vibration analysis of adhesive joints with functionally graded plate. By employing similar approaches, Apalak et al. (2006, 2014) investigated the free vibration of an adhesively bonded corner joint with single support and an adhesively bonded functionally graded double containment cantilever joint. The influence of vibration fatigue on the dynamic characteristics of adhesive joints was researched by Du and Shi (2014) through FEM and experiments. Based upon free vibration analysis, Paygozar and Sadigh (2017) applied FEM and ANNs to detect the cracks in adhesively bonded double-strap joints. Although FEM can well describe the free vibration behaviors of adhesive joints, yet cumbersome and time consuming modeling procedure including mesh generation, numerical calculation and post process is required. The FEM is not suitable for parametric study and optimization design owing to the need of regenerating mesh when varying the model geometrical parameters.

To simplify the spatial finite element mesh generation and efficiently model the adhesively bonded joints, a layerwise finite element was extended and employed in the paper for the purpose of transforming the spatial modeling problem to a 2D modeling problem. The joint can be regarded as a special multi-layer laminate by applying virtual boundary conditions. For modeling the multi-layer structure with viscoelastic adhesive layer, some layerwise finite 
element models have been reported. A beam element using discrete layer model was presented by Zapfe and Lesieutre (1999) for estimating the modal frequencies and damping of laminated beams with viscoelastic damping layers. Moreira and Rodrigues (2006a) and Moreira et al. (2006b) proposed a layerwise model to analyze sandwich plates with thin soft cores, and they compared the results with those obtained by traditional modeling method using a combination of standard solid and plate finite elements. By employing a mixed layerwise approach, a finite element model, which takes both the first-order shear theory and high-order shear theory into account, was developed by Araújo et al. (2010) for improving the dynamic performance of composite plates. Liu et al. (2017) used Carrera's Unified Formulation to formulate a layerwise finite element model, which can obtain high accurate results with fewer degrees of freedom, to analyze the dynamic behaviors of viscoelastic laminates. Xu et al. (2015) optimized viscoelastic damping structures by adopting a layerwise model. For the vibration analysis of sandwich plates with thick soft core, a layerwise element considering the compressive and extensive deformations in the viscoelastic core was presented by Ren et al. (2019). Despite that the layerwise model provides an efficient approach for modeling the joints, it still involves much time for numerical calculations. Therefore, ANNs were adopted to substitute the layerwise model for parametric study and optimization design.

For the current study, a layerwise plate finite element was extended for modeling the adhesive double-strap joint and then it was validated by three-dimensional solid finite element model. The model was subsequently applied to generate sampling points for training ANNs. With aid of the trained ANNs, parametric investigations considering the effects of the adhesive material properties and joint geometrical parameters on the vibration characteristics were performed. To lower the joint mass and improve the joint dynamic performance, a multiobjective optimization based upon nondominated sorting genetic algorithm was carried out to find optimal adhesive material properties and joint dimensions. Results indicate that the predicted natural frequencies and loss factors using the layerwise plate finite element are in good agreement with those obtained by threedimensional finite element model. Moreover, the ANNs combined with genetic algorithm can successfully deal with the multi-objective optimization problem. The proposed method provides a computationally efficient alternative for analyzing and optimizing the adhesive double-strap joint.

\section{Plate layerwise formulation}

Actually, similar layerwise finite element has been employed by Xu et al. (2015) to analyze plates with damping treatments. However, it was not used to describe the dynamic behaviors of adhesive joints. In a recent study (Wang et al., 2019), the layerwise plate finite element was applied by the present authors to model a stepped-lap joint and a singlelap joint. Nevertheless, its accuracy in the analysis of double-strap joints hasn't been validated. Besides, the optimal design based on the approach hasn't been carried out before. Below is a brief description of the plate layerwise formulation, and the double-strap joint was utilized as a typical example to formulate the plate layerwise element.
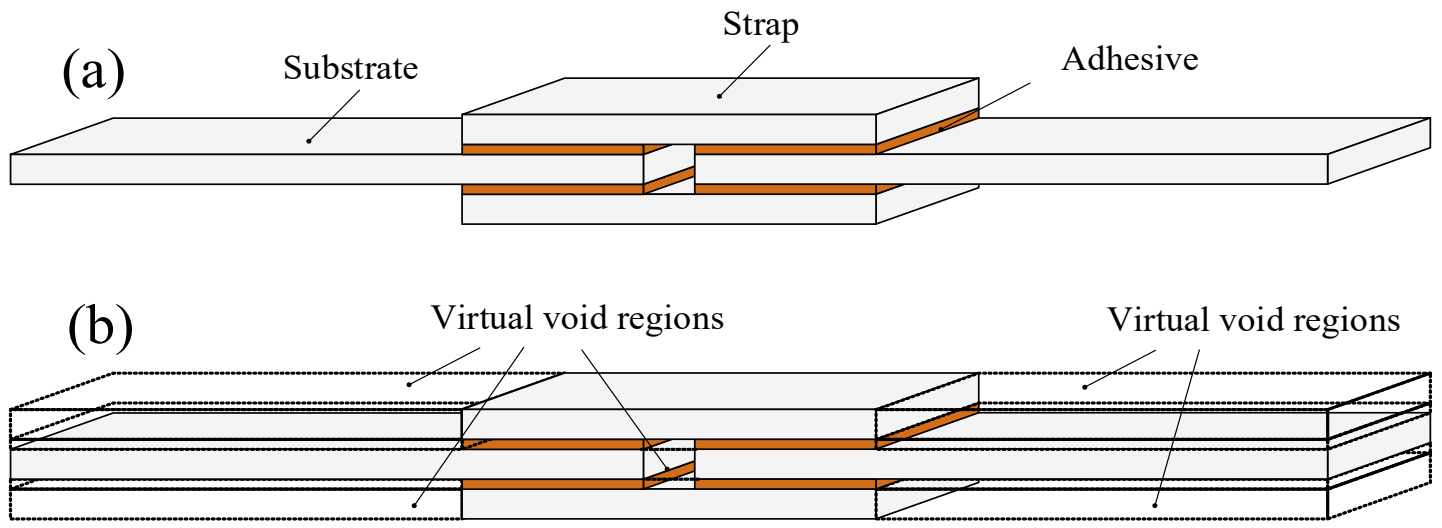

Fig. 1 Configuration of: (a) an adhesively bonded double-strap joint and (b) the equivalent multi-layer laminate plate. 


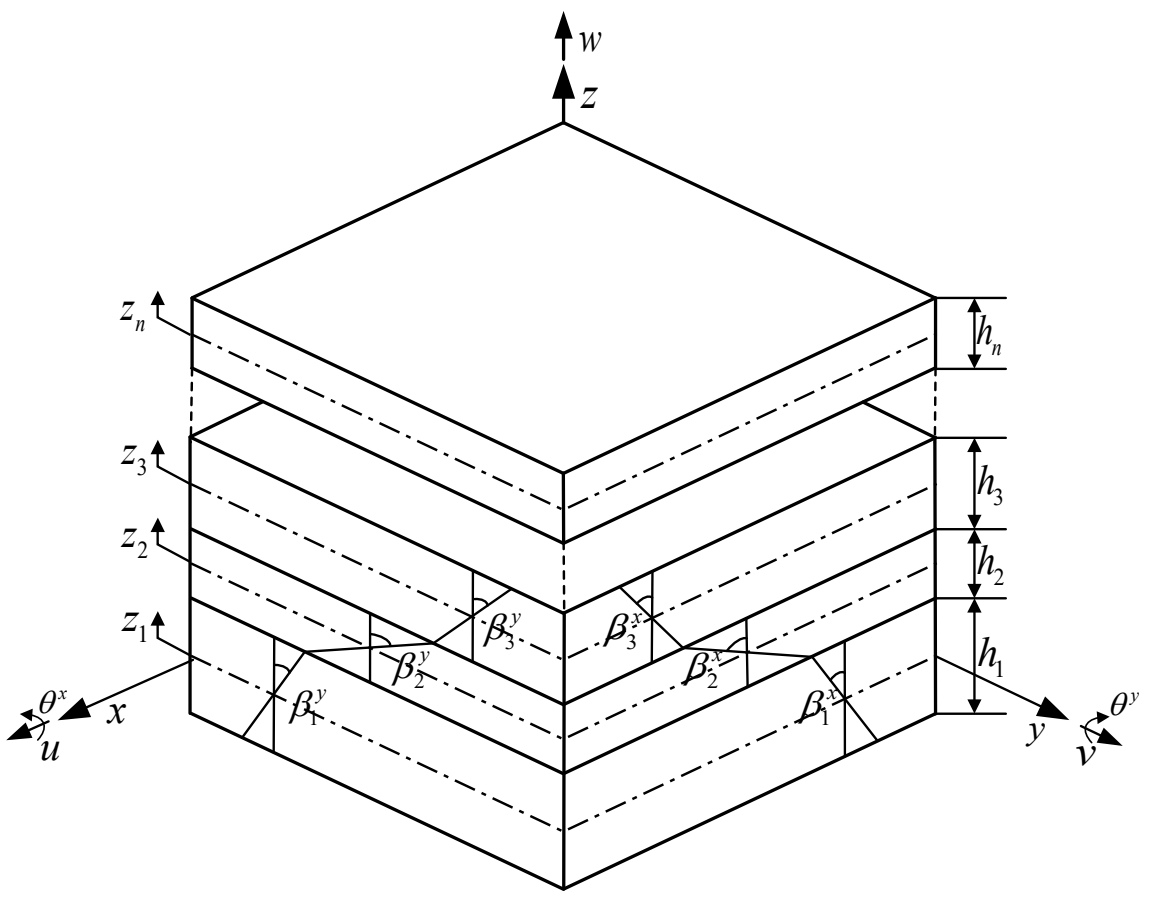

Fig. 2 Schematic view of displacement field of the multi-layer structure.

\subsection{Displacement field}

As illustrated in Fig. 1(a), the joint consists of two primary substrates, adhesive layers and two constraining straps (the substrates and straps can be seen as adherends). It is virtually filled and can be equivalent to a multi-layer laminate (Fig. 1(b)). As shown in Fig. 2, the multi-layer laminate is divided into $n$ layers along the thickness direction. Since the relatively thin layer is concerned with in the study, the deformation field in each layer is based upon first-order shear deformation theory (FSDT). For a generic $k$ th layer of the laminated plate, the related domain is defined as

$\Omega_{k}=\left\{\left(x, y, z_{k}\right) \in \mathrm{R}^{3} \mid z_{k} \in\left[-h_{k} / 2, h_{k} / 2\right],(x, y) \in A \subset \mathrm{R}^{2}\right\}$

where $\Omega_{k}, A$ and $h_{k}$ represent the volume, area and thickness of the $k$ th layer, respectively, $z_{k}$ is the local coordinate in thickness direction. The reference plane is the middle surface of the first layer $(k=1)$, and the displacement of other points in the laminate is determined with reference to its displacement. In order to obtain the displacement field in each layer $(k \geq 2)$, an interpolation matrix for a point in the $k$ th layer should be derived. As mentioned before, the displacement in each layer satisfies FSDT theory, so the in-plane displacement exhibits a zigzag pattern along thickness direction. In addition, displacement continuous conditions at layer interface should also be taken into account. Therefore, the displacement of each point in upper layer consists of two parts: one part is the displacement of the reference point on the reference plane and another is the displacement caused by zigzag effects. The displacement of a point in the $k$ th layer can be expressed as

$\{\mathbf{u}\}_{k}=\left\{\begin{array}{l}u_{k} \\ v_{k} \\ w_{k}\end{array}\right\}=\left\{\begin{array}{c}u_{0}+\frac{h_{1}}{2} \beta_{1}^{x}+\sum_{j=2}^{k-1} h_{j} \beta_{j}^{x}+\frac{h_{k}}{2} \beta_{k}^{x}+z_{k} \beta_{k}^{x} \\ v_{0}+\frac{h_{1}}{2} \beta_{1}^{y}+\sum_{j=2}^{k-1} h_{j} \beta_{j}^{y}+\frac{h_{k}}{2} \beta_{k}^{y}+z_{k} \beta_{k}^{y} \\ w_{0}\end{array}\right\}$

where $u_{0}, v_{0}$ and $w_{0}$ are the translations of a reference point on the reference plane, and $\beta^{y}, \beta^{x}\left(\beta^{y}=-\theta^{x}, \beta^{x}=\theta^{y}\right)$ denote the rotations of the normal about the $x$ - and y-axes, respectively. Eq. (2) can be written into a matrix form 
$\{\mathbf{u}\}_{k}=\left\{\begin{array}{lll}u_{k} & v_{k} & w_{k}\end{array}\right\}^{\mathrm{T}}=[\hat{N}]_{k}\{d\}$

where $[\hat{N}]_{k}$ represents the interpolation matrix, and $\{d\}$ is the generalized displacement field. Specifically,

$[\widehat{N}]_{k}=\left[\begin{array}{cccccccccccc}1 & 0 & 0 & h_{1} / 2 & 0 & \cdots & h_{j} & 0 & \cdots & h_{k} / 2+z_{k} & 0 & \\ 0 & 1 & 0 & 0 & h_{1} / 2 & \cdots & 0 & h_{j} & \cdots & 0 & h_{k} / 2+z_{k} & {[0]} \\ 0 & 0 & 1 & 0 & 0 & \cdots & 0 & 0 & \cdots & 0 & 0 & \end{array}\right]$

and

$\{d\}=\left\{u_{0}, v_{0}, w_{0}, \beta_{1}^{x}, \beta_{1}^{y}, \ldots . \beta_{k}^{x}, \beta_{k}^{y}, \ldots \ldots \beta_{n}^{x}, \beta_{n}^{y}\right\}^{\mathrm{T}}$

\subsection{Strain and stress fields}

Based on the displacement field, the strain field of the $k$ th layer can be obtained through applying a differential operator matrix $[L]$

$\{\varepsilon\}_{k}=[L]\{\mathbf{u}\}_{k}$

$[L]=\left[\begin{array}{ccc}\frac{\partial}{\partial x} & 0 & 0 \\ 0 & \frac{\partial}{\partial y} & 0 \\ \frac{\partial}{\partial y} & \frac{\partial}{\partial x} & 0 \\ \frac{\partial}{\partial z} & 0 & \frac{\partial}{\partial x} \\ 0 & \frac{\partial}{\partial z} & \frac{\partial}{\partial y}\end{array}\right]$

Then, substituting Eq. (3) into Eq. (6) yields

$\{\varepsilon\}_{k}=[L][\hat{N}]_{k}\{d\}=[B]_{k}\{d\}$

where $[B]_{k}$ is the deformation matrix of the $k$ th layer.

The materials of the plate are assumed being made of linearly elastic materials. In this case, the constitutive equation for the $k$ th layer can be defined as

$\{\sigma\}_{k}=[D]_{k}\{\varepsilon\}_{k}=\operatorname{diag}\left([D]_{k}^{I},[G]_{k}^{B}\right)\left\{\{\varepsilon\}_{k}^{I} ;\{\varepsilon\}_{k}^{B}\right\}^{\mathrm{T}}$

where the superscripts I denotes the in-plane deformation terms including membrane, coupling and in-plane shear deformations, while $B$ denotes the out-plane bending term. For isotropic or orthotropic materials, $[D]_{k}$ can be specifically expressed as 


$$
\left\{\begin{array}{l}
\sigma_{11} \\
\sigma_{22} \\
\sigma_{12} \\
\sigma_{13} \\
\sigma_{23}
\end{array}\right\}_{k}=\left[\begin{array}{ccccc}
D_{11} & D_{12} & D_{14} & 0 & 0 \\
& D_{22} & D_{24} & 0 & 0 \\
& & D_{44} & 0 & 0 \\
& & & D_{55} & D_{56} \\
\text { sym. } & & & & D_{66}
\end{array}\right]_{k}\left\{\begin{array}{l}
\varepsilon_{11} \\
\varepsilon_{22} \\
\varepsilon_{12} \\
\varepsilon_{13} \\
\varepsilon_{23}
\end{array}\right\}_{k}
$$

In most cases, $D_{i j}$ is a real number. However, for viscoelastic materials, $D_{i j}$ is taken to be complex. As the adhesive layer is assumed to have a viscoelastic behavior in the study, the final stiffness matrix would be a complex matrix. The detailed form of constitutive matrix can be found in (Jones, 2014). Moreover, it should be noted that the deformation matrix, $[B]_{k}$, is accordingly divided into two sub-matrices

$$
\left\{[B]_{k}\right\}_{5 \times(2 n+3)}=\left\{\begin{array}{l}
\left\{[B]_{k}^{I}\right\}_{3 \times(2 n+3)} \\
\left\{[B]_{k}^{B}\right\}_{2 \times(2 n+3)}
\end{array}\right\}
$$

\subsection{Potential and kinetic energy}

The potential energy of the laminated plate can be achieved by the sum of the potential energy of each layer. More precisely,

$$
\Pi^{P}=\sum_{k=1}^{n} \frac{1}{2} \int_{\Omega_{k}}\{\varepsilon\}_{k}^{\mathrm{T}}\{\sigma\}_{k} \mathrm{~d} \Omega_{k}=\sum_{k=1}^{n} \frac{1}{2} \int_{\Omega_{k}}\{d\}^{\mathrm{T}}[B]_{k}^{\mathrm{T}}[D]_{k}[B]_{k}\{d\} \mathrm{d} \Omega_{k}
$$

By the similar way, the kinetic energy of the laminated plate can be obtained by the sum of the kinetic energy of each layer. The velocity field of a generic $k$ th layer can be achieved from the time derivative of the generalized displacement field. Hence, the kinetic energy of the laminated plate can be expressed as

$\Pi^{K}=\sum_{k=1}^{n} \frac{1}{2} \rho_{k} \int_{\Omega_{k}}\{\dot{\mathbf{u}}\}_{k}^{\mathrm{T}}\{\dot{\mathbf{u}}\}_{k} \mathrm{~d} \Omega_{k}=\sum_{k=1}^{n} \frac{1}{2} \rho_{k} \int_{A} \int_{z_{k}}\{\dot{d}\}_{k}^{\mathrm{T}}[\hat{N}]_{k}^{\mathrm{T}}[\hat{N}]_{k}\{\dot{d}\}_{k} \mathrm{~d} z_{k} \mathrm{~d} A$

where $\rho_{k}$ is the mass density of $k$ th layer. Eq. (13) can be further rearranged as follows

$\Pi^{K}=\sum_{k=1}^{n} \frac{1}{2} \int_{A}\{\dot{d}\}_{k}^{\mathrm{T}}[J]_{k}\{\dot{d}\}_{k} \mathrm{~d} A$

where $[J]_{k}=\int_{z_{k}} \rho_{k}[\hat{N}]_{k}^{\mathrm{T}}[\hat{N}]_{k} \mathrm{~d} z_{k}$ can be seen as an inertia matrix.

\subsection{Interpolation and discretization}

An isoparametric quadrangular finite element with four nodes is applied to model the plate, in which bilinear shape functions are chosen to interpolate the geometry and displacement field within the finite element domain. The shape functions are defined as

$N_{i}(\xi, \eta)=\frac{1}{4}\left(1+\xi_{i} \xi\right)\left(1+\eta_{i} \eta\right) \quad i=1,2,3,4$

where $\xi$ and $\eta$ are local coordinates. The next is to derive the element stiffness matrix [ $\left.K^{e}\right]$ and the element mass matrix $\left[M^{e}\right]$. Applying the Hamilton's principle to Eqs. (12) and (13), $\left[K^{e}\right]$ and $\left[M^{e}\right]$ can finally be given as 


$$
\begin{aligned}
& {\left[K^{e}\right]=\sum_{k=1}^{n}\left[K^{e}\right]_{k}=\sum_{k=1}^{n} \int_{\Omega_{k}^{e}}\left[B^{e}\right]_{k}^{\mathrm{T}}\left[D^{e}\right]_{k}\left[B^{e}\right]_{k} \mathrm{~d} \Omega_{k}^{e}} \\
& {\left[M^{e}\right]=\sum_{k=1}^{n}\left[M^{e}\right]_{k}=\sum_{k=1}^{n} \int_{\Omega^{e}}\left[N^{e}\right]^{\mathrm{T}}\left[J^{e}\right]_{k}\left[N^{e}\right] \mathrm{d} \Omega_{k}^{e}}
\end{aligned}
$$

where $\left[N^{e}\right]$ is the shape function matrix of the finite element, $\left[B^{e}\right]_{k}$ is the deformation matrix and can be calculated by $[B]_{k}\left[N^{e}\right]$. For the sake of convenient computation, Eq. (11) is substituted into $[B]_{k}\left[N^{e}\right]$ and $\left[B^{e}\right]_{k}$ is accordingly divided into two sub-matrices. Hence, $\left[K^{e}\right]_{k}$ is separated into two parts.

$\left[K^{e}\right]_{k}=\int_{\Omega_{k}^{e}}\left[B^{e}\right]_{k}^{I \mathrm{~T}}\left[D^{e}\right]_{k}\left[B^{e}\right]_{k}^{I} \mathrm{~d} \Omega_{k}^{e}+\int_{\Omega_{k}^{e}}\left[B^{e}\right]_{k}^{B \mathrm{~T}}\left[D^{e}\right]_{k}\left[B^{e}\right]_{k}^{B} \mathrm{~d} \Omega_{k}^{e}=\left[K^{e}\right]_{k}^{I}+\left[K^{e}\right]_{k}^{B}$

Note that the joint structure is a thin quasi-sandwich plate in most cases. Therefore, the transverse shear locking must be concerned about. One approach to address the issue is imposing reduced integration on the bending component $\left(\left[K^{e}\right]_{k}^{B}\right)$ of the stiffness formulation.

Finally, the total $n_{E}$ elemental mass matrices and $n_{E}$ stiffness matrices can be assembled into global mass matrix, $[M]$, and stiffness matrix, $[K]$, respectively. And the global finite element equations of motion for the free vibration problem is given by

$$
[M]\{\ddot{d}\}+[K]\{d\}=\{0\}
$$

\subsection{Equivalent boundary conditions}

As aforementioned, the joint structure can be viewed as a special multi-layer structure which exists some voids. As shown in Fig. 1(b), the void regions are denoted as $\Omega_{V}$. Specifically in a layerwise finite element model for a joint, the void parts are involved and meshed as a whole plate, of which, however, the mechanical effects are ignored. In other words, the elements located in $\Omega_{V}$ have no contribution to the stiffness and mass matrix. To simulate this operation, the thicknesses and densities of the layers in $\Omega_{V}$ are particularly set zero, i.e.,

$$
h_{k}^{e}=\rho_{k}^{e}=0\left(e, k \in \Omega_{V}\right)
$$

On the other hand, the rotational degrees of freedom in these regions should also be restricted to accommodate the above zero-setting operations, otherwise, the stiffness matrix will become singular.

$\beta^{x}=\beta^{y}=0\left(e, k \in \Omega_{V}\right)$

For a specific harmonic vibration problem, Eq. (19) will finally reduce to Eq. (22) after the actual boundary conditions are employed, which is a typical eigenvalue problem and could be resolved through the Lanczos algorithm (Lehoucq and Sorensen, 1996)

$$
\left([\widehat{K}]-\lambda_{r}^{*}[\widehat{M}]\right)\left\{\phi^{*}\right\}=\{0\}
$$

Noting that the components of joints are allowed to be viscoelastic materials, the stiffness matrix can be a complex one, and thus $\left\{\phi^{*}\right\}$ and $\lambda_{r}^{*}$ are complex eigenvectors and eigenvalues, respectively. The form of $\lambda_{r}^{*}$ can be further expressed as 


$$
\lambda_{r}^{*}=\lambda_{r}\left(1+i \eta_{r}\right)
$$

where $\eta_{r}$ is the modal loss factor of $r$-th mode. And the natural frequency for $r$-th mode is

$$
f_{r}=\frac{\sqrt{\lambda_{r}}}{2 \pi}
$$

\section{Validation}

The commercial finite element package ABAQUS was utilized to validate the accuracy of the proposed layerwise plate element model. The geometry of the double-strap joint under investigation is illustrated in Fig. 3 . Two primary substrates are joined by upper and lower constraining straps using epoxy adhesive layers. All degrees of freedom at the left end of the joint are fixed, in accord with the clamped boundary condition. The materials of substrates and straps are selected as aluminum alloy, the mechanical properties of which were as follows: Young's modulus $E=69$ GPa , Poisson's ratio $v=0.33$ and a density $\rho=2800 \mathrm{~kg} / \mathrm{m}^{3}$. The adhesive layer is assumed being made of a kind of epoxy adhesive, which has a Young's modulus $E_{a}=4.39 \mathrm{GPa}$, Poisson's ratio $v_{a}=0.34$ and a density $\rho_{a}=2500 \mathrm{~kg} / \mathrm{m}^{3}$. The viscoelastic property of the adhesive is considered in this work, and the adhesive loss factor is assumed to be 0.1 . In addition, as shown in Fig. 3, the joint has an overall length of $260 \mathrm{~mm}$ and a width of $40 \mathrm{~mm}$, among which the overlap length, $L_{\mathrm{o}}$, is $60 \mathrm{~mm}$. Other parameters including the adhesive thickness and the substrates thickness are $0.5 \mathrm{~mm}$ and $4 \mathrm{~mm}$, respectively. Moreover, the thickness of straps is half of substrates and the length of the gap between the substrates is $1 \mathrm{~mm}$.

An eight node linear brick element C3D8R characterized by reduced integration and hourglass control was used to model the substrates, straps and adhesive layers in ABAQUS. Since the thickness of adhesive layer is smaller than that of the substrates and straps, the finite element mesh in adhesive layer was refined in order to obtain an accurate indication of the joint dynamic responses. Mesh convergence analysis has been performed to ensure that increasing the number of elements has minor effects on the results. The mesh configurations of the presented layerwise plate model and the comparative model are shown in Fig. 4.

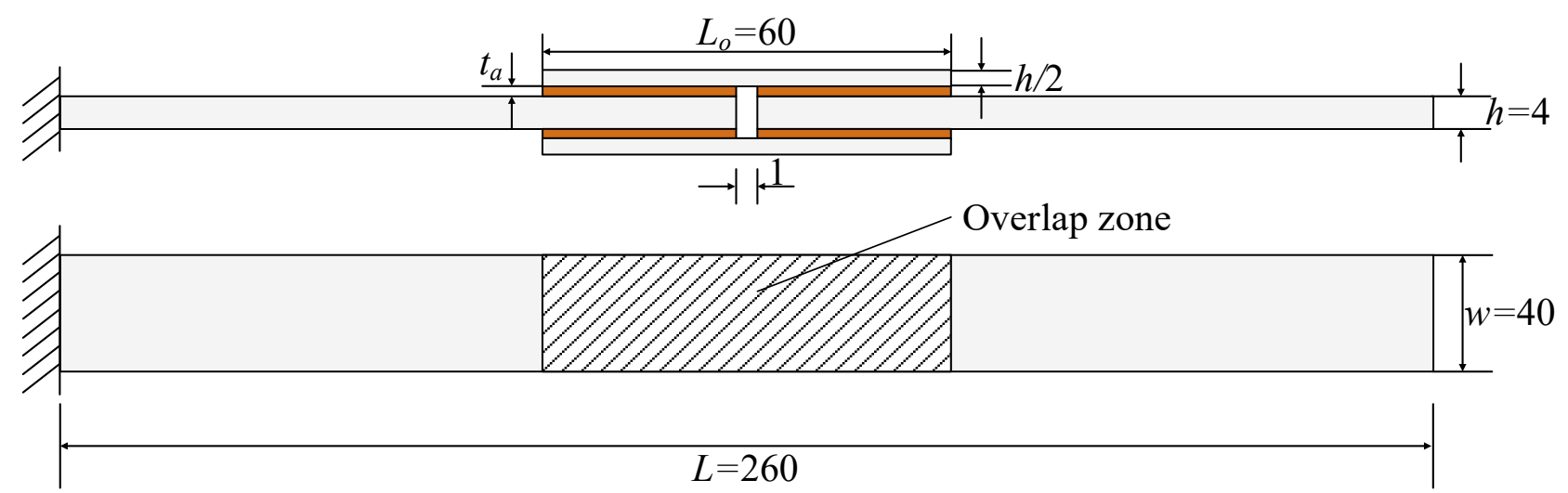

Fig. 3 Dimensions of an adhesively bonded double-strap joint (unit in $\mathrm{mm}$ ). 


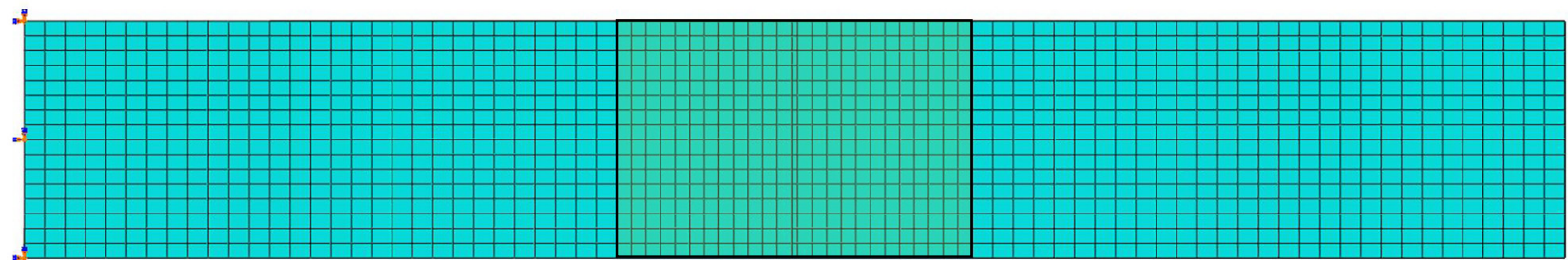

(a)
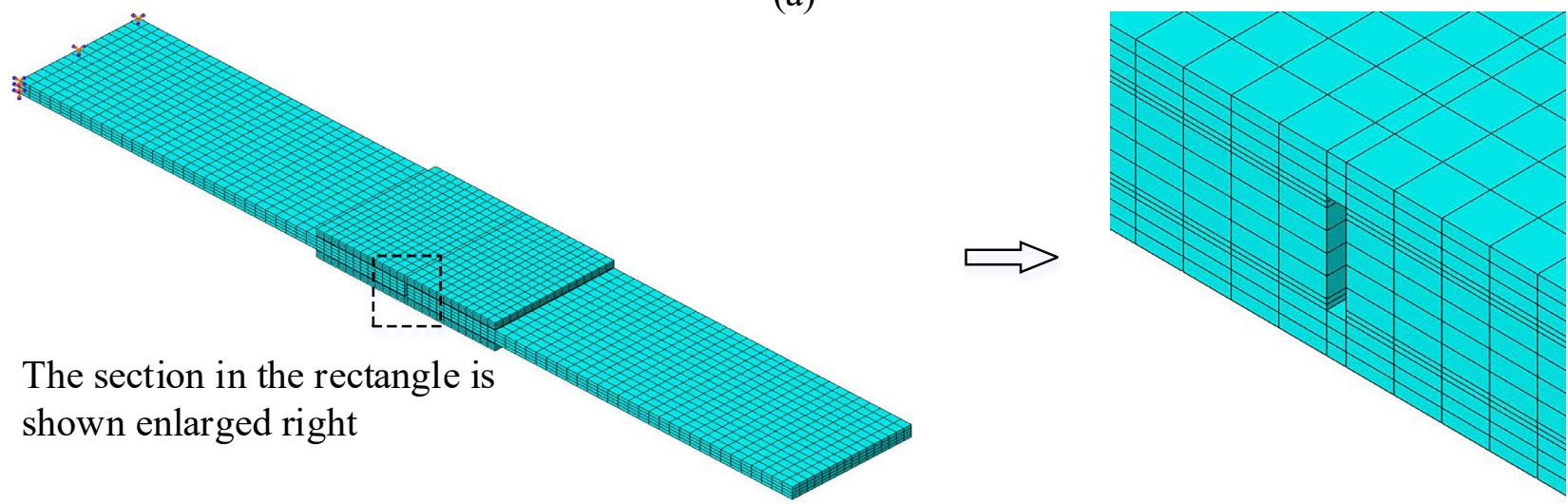

(b)

Fig. 4 Meshes for (a) the present layerwise element model; (b) the solid finite element model.

The first ten natural frequencies and corresponding loss factors obtained by FEM and the proposed layerwise element model are listed in Table 1, from which we find that the joint tends to bending modes, and the lateral modes are relatively rare, for five bending modes and three torsional modes occur but only two lateral modes exist in the first ten modes. Furthermore, it can be noticed that the present numerical results are in good agreement with those obtained by three-dimensional finite element model, except for some discrepancies in lateral vibrations. As we can see, the modal loss factors from FEM and the present approach don't agree very well for the lateral modes (mode 3 and 8 ). However, the natural frequencies of the lateral modes are still relatively accurate. Actually, regardless of analytical solutions or finite element analysis, little attention is given to the joint lateral vibrations, as we found the previous related researches almost concentrated on the longitudinal, torsional or bending (transverse) vibration behaviors. Besides, when we vary the joint geometry, the lateral mode can be so rare that only one or none exists in the first ten modes. Therefore, the inaccuracy in the calculation on modal loss factors of lateral vibrations can be ignored. It should also be mentioned that the joint may experience longitudinal modes in the first ten modes, and the present method can predict both the natural frequency and loss factor accurately (not shown in the paper). The comparison has proven that the present model is good enough to analyze the joint vibration characteristics.

Table 1 Comparison of results obtained by FEM and the present layerwise model.

\begin{tabular}{|c|c|c|c|c|}
\hline & \multicolumn{2}{|c|}{ FEM } & \multicolumn{2}{|c|}{ Present } \\
\hline & $f_{r} / \mathrm{Hz}$ & $\eta_{r} / \%$ & $f_{r} / \mathrm{Hz}$ & $\eta_{r} / \%$ \\
\hline 1 (B\#1) & 47.04 & 0.034 & 46.96 & 0.036 \\
\hline $2(\mathrm{B \#})$ & 335.22 & 0.235 & 332.80 & 0.247 \\
\hline 3 (L\#1) & 440.96 & $\underline{0.173}$ & 443.63 & $\underline{0.075}$ \\
\hline 4 (T\#1) & 570.78 & 0.114 & 578.02 & 0.125 \\
\hline 5 (B\#3) & 844.22 & 0.105 & 808.15 & 0.107 \\
\hline $6(\mathrm{~T} \# 2)$ & 1785.48 & 0.166 & 1807.60 & 0.176 \\
\hline 7 (B\#4) & 2059.52 & 0.267 & 1988.75 & 0.275 \\
\hline $8(\mathrm{L \# 2})$ & 2273.75 & $\underline{0.623}$ & 2294.40 & $\underline{0.375}$ \\
\hline 9 (B\#5) & 2901.60 & 0.270 & 2859.12 & 0.259 \\
\hline 10 (T\#3) & 3585.71 & 0.281 & 3546.76 & 0.274 \\
\hline
\end{tabular}

Note: B\#i, i-th bending mode; L\#i, i-th lateral mode; T\#i, i-th torsional mode. 
Although the layerwise plate finite element is able to describe the dynamic behaviors of the adhesive joint efficiently, it is still not suitable for parametric study and optimization design owing to time-consuming numerical calculation. In order to deal with the problem, ANNs were established to predict the natural frequency and loss factor of the double-strap joint. In the following section, several ANNs will be developed, by which the parametric investigation and optimization design will be carried out thereafter.

\section{ANN model}

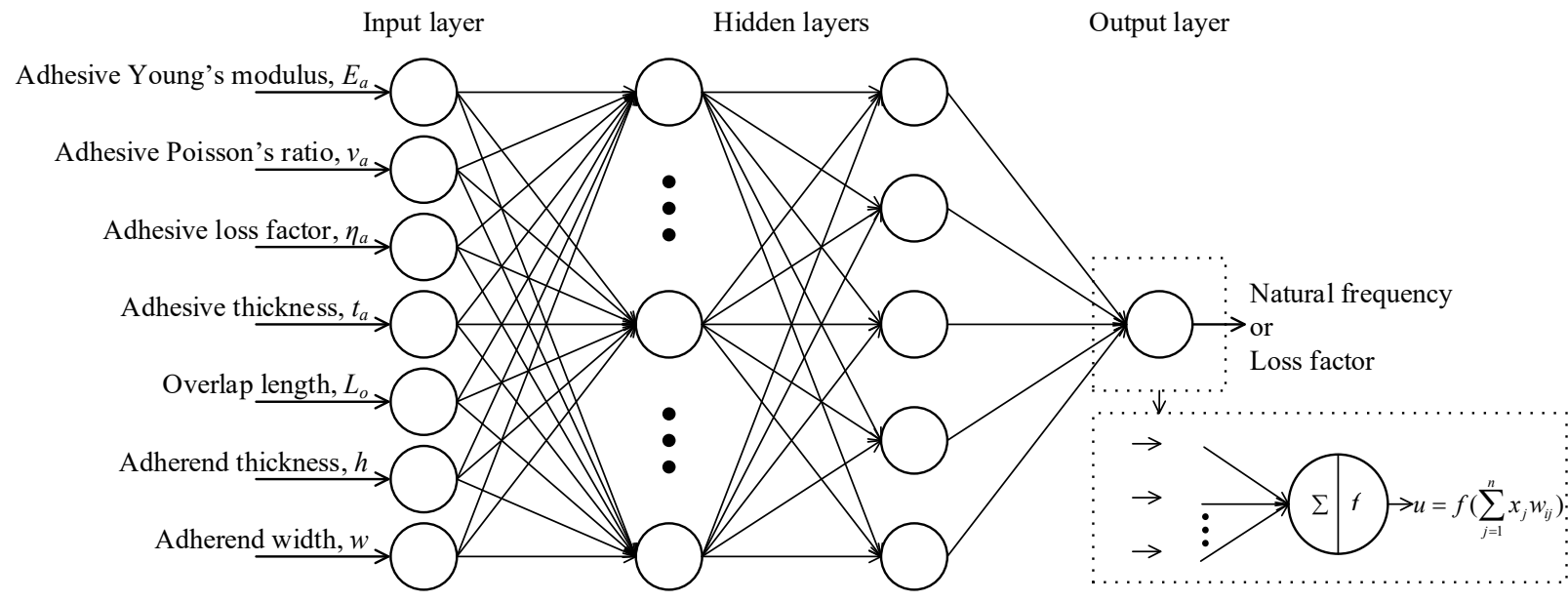

Fig. 5 Architecture of multilayer back-propagation neural network.

As illustrated in Fig. 5, an ANN can be seen as a nonlinear mapping between the input space and output space. The basic element constituting this neural network is a neuron, which is actually an information processing unit simulating the behavior and function of a biological neuron. A neuron receives weighted inputs from other neurons through its incoming connections. The weighted inputs are summed and then the outcome is taken as input for the activation function to form the neuron output. Network topology with one input layer ( 7 neurons take on the values of input variables), two hidden layers ( 20 and 5 neurons respectively) and one output layer was chosen for the study. The sigmoid function which has the form $f(n)=1 /[1+\exp (-n)]$ and the linear function which has the form $f(n)=n$ were chosen as activation functions for the hidden layers and output layer, respectively. The networks were trained by LevenbergMarquardt algorithm, which can achieve second-order training speed and converge quickly.

The inputs of the network include adhesive material properties such as Young's modulus $E_{a}$, Poisson's ratio $v_{a}$, material loss factor $\eta_{a}$, and joint geometrical parameters such as adhesive thickness $t_{a}$, overlap length $L_{o}$, adherend thickness $h$ and width $w$. The overall length of $260 \mathrm{~mm}$ is kept constant throughout the analysis. The ranges of the design parameters are set as follows: $1 \mathrm{GPa} \leq E_{a} \leq 10 \mathrm{GPa}, \quad 0.3 \leq v_{a} \leq 0.5, \quad 0 \leq \eta_{\mathrm{a}} \leq 5, \quad 0.05 \mathrm{~mm} \leq t_{\mathrm{a}} \leq 1.0 \mathrm{~mm}$, $20 \mathrm{~mm} \leq L_{\mathrm{o}} \leq 150 \mathrm{~mm}, 2 \mathrm{~mm} \leq h \leq 6 \mathrm{~mm}$ and $20 \mathrm{~mm} \leq w \leq 60 \mathrm{~mm}$. The range of adhesive properties covers the mechanical properties of various types of structural adhesive including rubbers, elastomers, epoxies and ceramic glues. The rubbers and elastomers have low values of Young's modulus (down to $0.001 \mathrm{GPa}$ ) and high values of Poisson's ratio (up to 0.5 ), whereas the ceramic glues have high values of Young's modulus (up to $10 \mathrm{GPa}$ ) and low values of Poisson's ratio (down to 0.3 ), meanwhile, the epoxies have intermediate values of Young's modulus and Poisson's ratio (He, 2014). As the adhesives with Young's modulus less than $1 \mathrm{GPa}$ are not commonly used in practice, the cases with Young's modulus less than $1 \mathrm{GPa}$ were not investigated.

To ensure that the variables are not clustered in any region of their respective ranges, an optimal Latin hypercube design (Morris and Mitchell, 1995) was utilized to generate 500 sampling points within the given ranges. Based on the sampling points, the layerwise finite element model was subsequently employed to obtain corresponding natural frequencies and loss factors to form training set. In addition, a randomly generated 40 patterns were used to attest the accuracy of the trained neural networks.

The neural network toolbox in Matlab was used to establish the proposed ANN models. The input and output training data was normalized between 0 and 1 to avoid numerical problems. Mean squared error (MSE, the average squared difference between outputs and targets) was adopted as performance function to train the networks. The MSE 
was decreased to $10^{-8}-10^{-5}$ during the training process. Among all the eigenvalues, the first natural frequency is important, since it has significant influence on the fatigue of the adhesive joints. Besides, resonance may occur when an external excitation has a frequency near the first natural frequency, resulting in the damage of the joint. Consequently, it is more meaningful to figure out the effects of the adhesive material properties and joint geometrical parameters on the vibration characteristics by considering the first natural frequency. For the first mode, two ANNs for the natural frequency and corresponding loss factor were established. The comparisons of the predicted results given by ANNs with the test data given by the present layerwise finite element model are illustrated in Fig. 6 . As we can see, the predicted results show good agreements with the corresponding testing results in both natural frequency and modal loss factor. Similar performances are also observed for other vibration modes, but omitted here. Such a good agreement proves the applicability of the present ANN model.
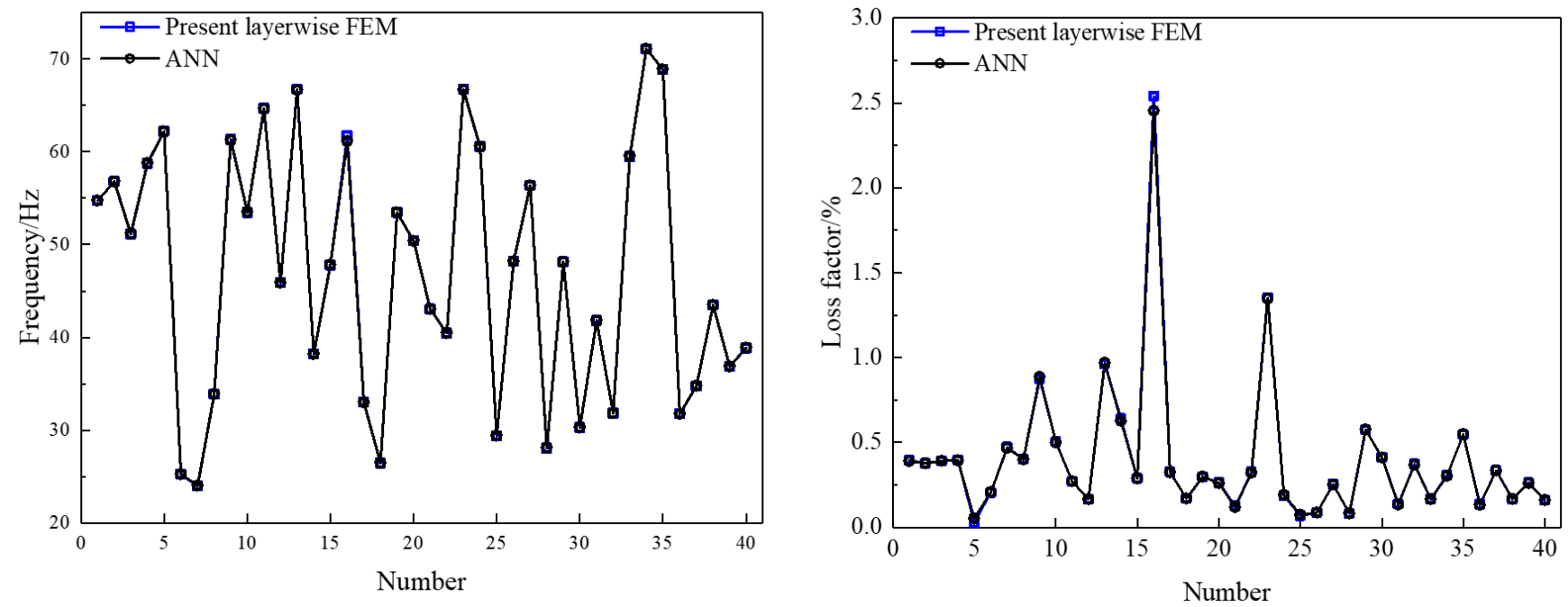

Fig. 6 The comparative results of natural frequency and loss factor obtained from the ANN and the present layerwise FEM.

\section{Parametric investigation}

Adhesively bonded joints are often subjected to dynamic loads or may experience an external excitation, which may lead to initiation and propagation of failure in the joint. To guarantee the joint reliability, it is essential to have a knowledge of the effects of various design variables on the joint dynamic responses. In the following, the modal frequency and loss factor of the first mode are investigated with aid of the established ANN model. The fundamental joint configuration has an adhesive thickness of $0.5 \mathrm{~mm}$, an adhesive loss factor of 0.2 , an adhesive Young's modulus of $2 \mathrm{GPa}$, an adhesive Poisson's ratio of 0.34 , an overlap length of $60 \mathrm{~mm}$, an adherend thickness of $4 \mathrm{~mm}$ and an adherend width of $30 \mathrm{~mm}$. When investigating the combined effects of a pair of variables, the values of other variables are equal to the values of fundamental joint configuration. The ranges of the parameters are provided in Section. 4.

The combined effects of the adhesive thickness $t_{a}$ with the adhesive loss factor $\eta_{a}$ on the first natural frequency and corresponding loss factor are shown in Fig. 7(a). For natural frequency, the result surface exhibits a gradual rise at low $\eta_{\mathrm{a}}$ stage, and turns to a flat plane at high $\eta_{\mathrm{a}}$ stage. The changing trend of modal loss factor with $\eta_{\mathrm{a}}$ is different from that of natural frequency. The increase of $\eta_{\mathrm{a}}$ results in a sharp increase of modal loss factor at the early stage, but turns to a slight drop then approaching a final constant at the later stage. In other words, blindly increasing the adhesive loss factor cannot always improve the joint damping performance. On the other hand, it can be found that the values of natural frequency and loss factor present nearly linear but opposite trends with $t_{\mathrm{a}}$ varying. From a low $t_{\mathrm{a}}$ to a high one, the natural frequency exhibits a dropping trend, while the modal loss factor shows an increasing trend. 

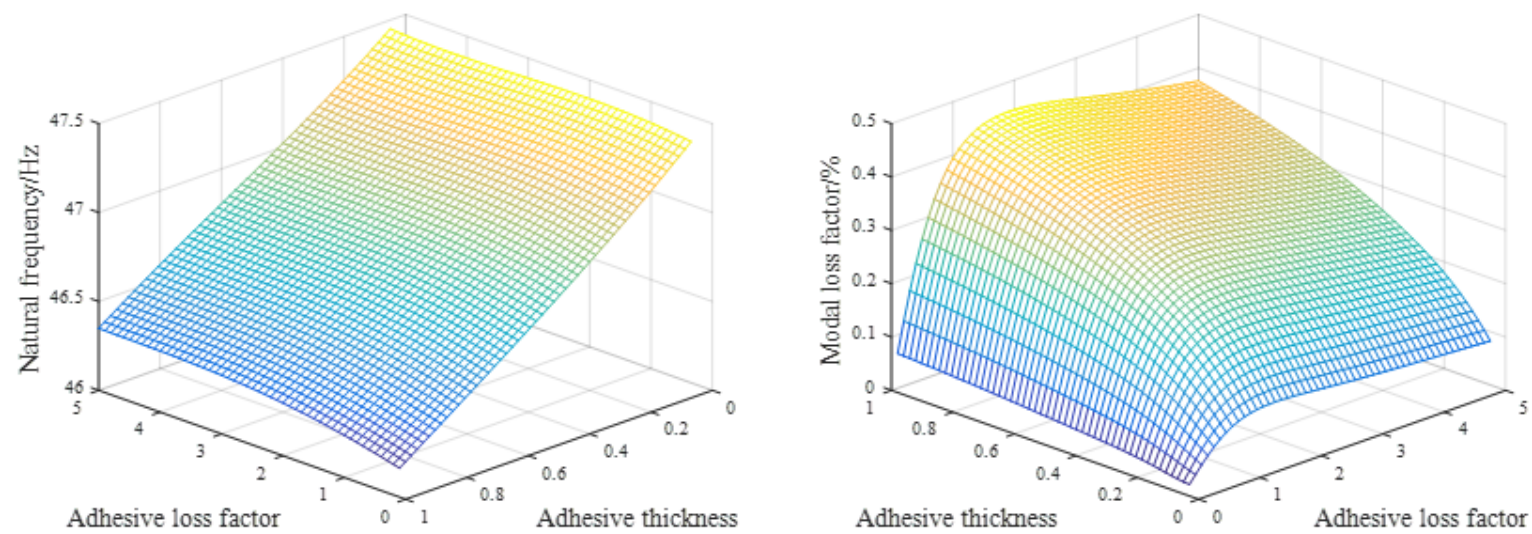

(a) Adhesive loss factor-Adhesive thickness
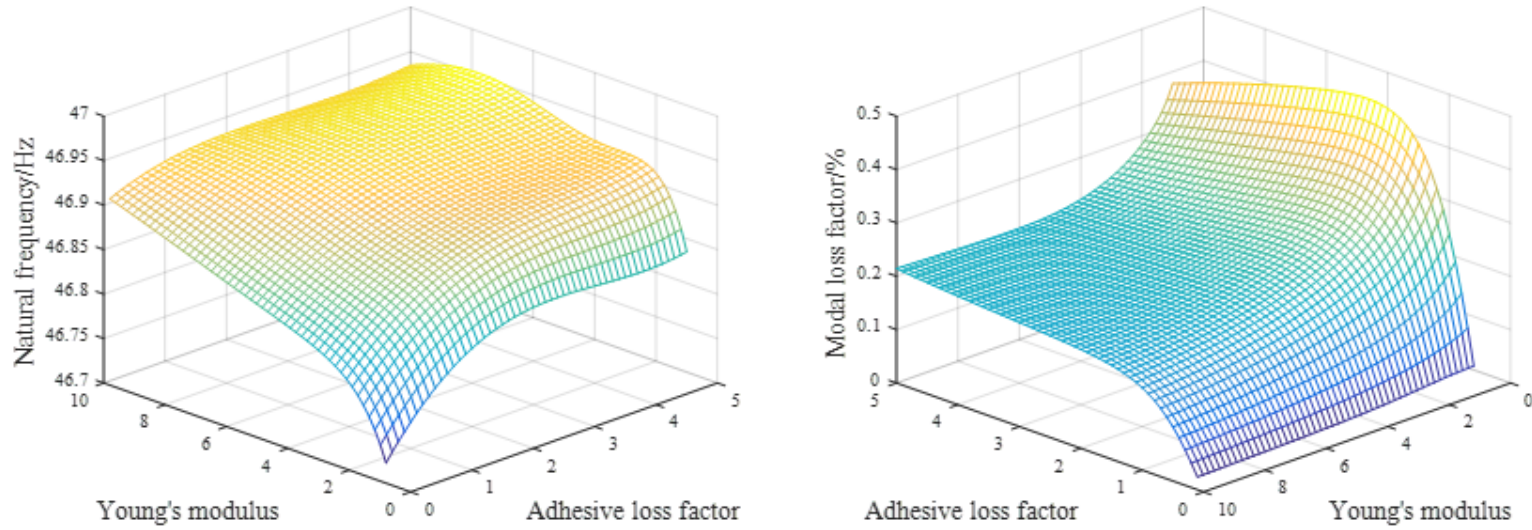

(b) Adhesive loss factor-Adhesive Young's modulus
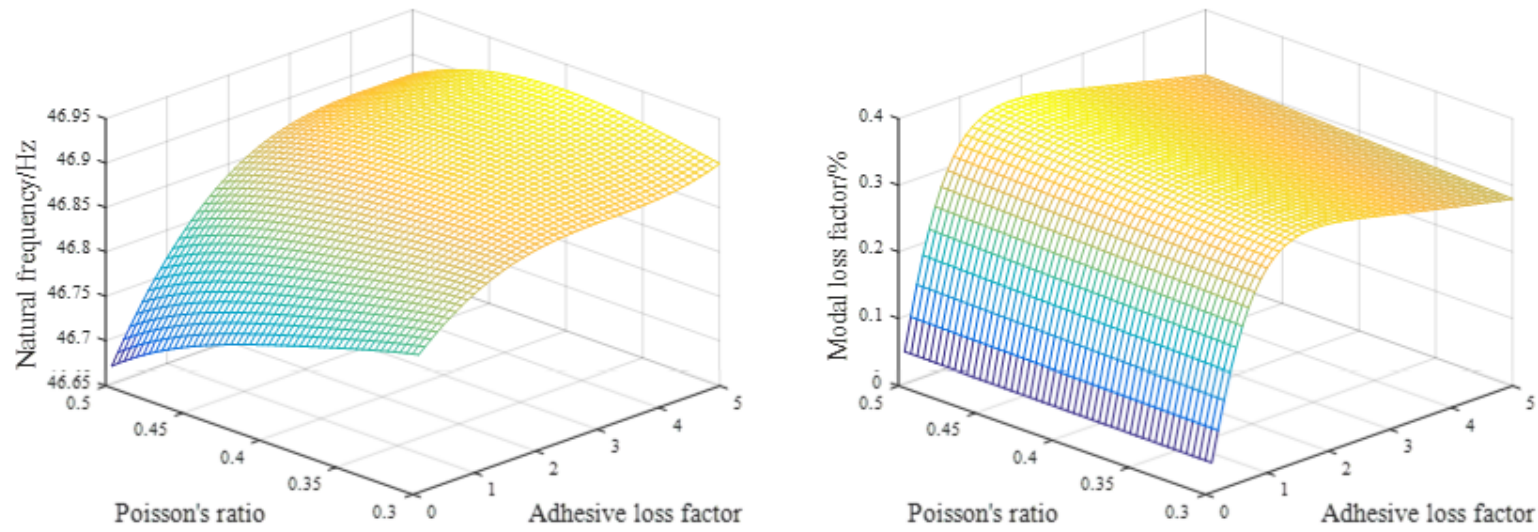

(c) Adhesive loss factor-Adhesive Poisson's ratio

Fig. 7 Combined effects of the adhesive layer.

Fig. 7(b) depicts the combined effects of adhesive loss factor and Young's modulus on the vibration properties. It can be noticed that the natural frequency tends to increase with increasing the adhesive Young's modulus, but the modal loss factor tends to decrease. Besides, the variation of natural frequency with adhesive Young's modulus and loss factor is in a relatively small range. Moreover, the effects of adhesive Young's modulus on modal loss factor are more evident when it is small (less than $2 \mathrm{GPa}$ ) and are minor when it is large (more than $6 \mathrm{GPa}$ ). As illustrated in Fig. 7(c), increasing adhesive Poisson's ratio appears to reduce the natural frequency, but the effect is negligible when the adhesive loss factor is large. The modal loss factor keeps almost constant with adhesive Poisson's ratio varying. Through the above analysis, the joint can achieve a relatively large loss factor using thick adhesive layer (e.g., $1 \mathrm{~mm}$ ) with small Young's 
modulus (e.g., $1 \mathrm{GPa}$ ) and appropriate loss factor (e.g., 2). On the contrary, the combinations of parameters would result in a relatively small natural frequency.
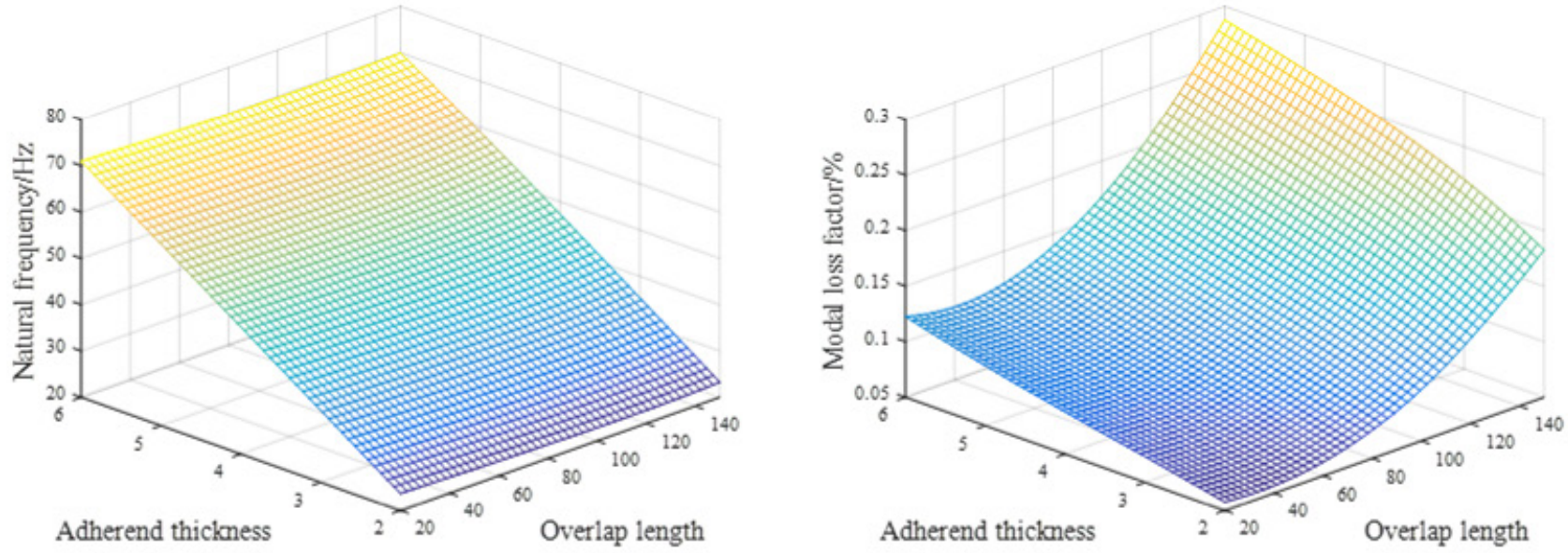

(a) Adherend thickness-Overlap length
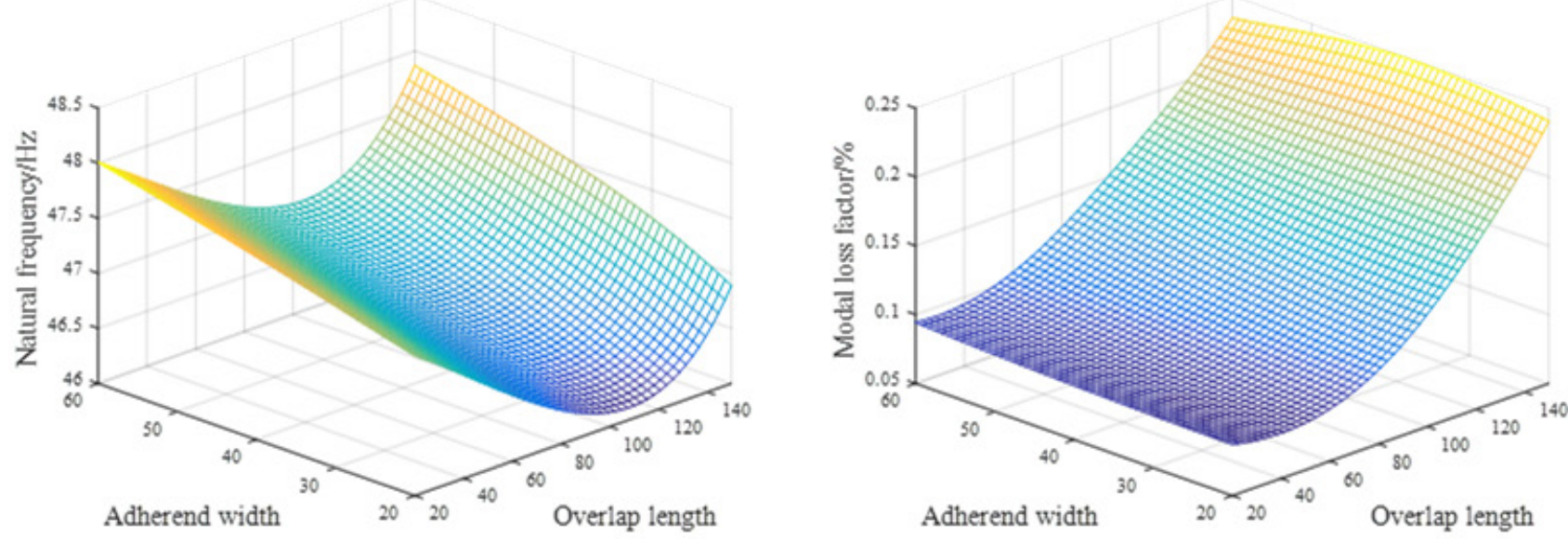

(b) Adherend width-Overlap length
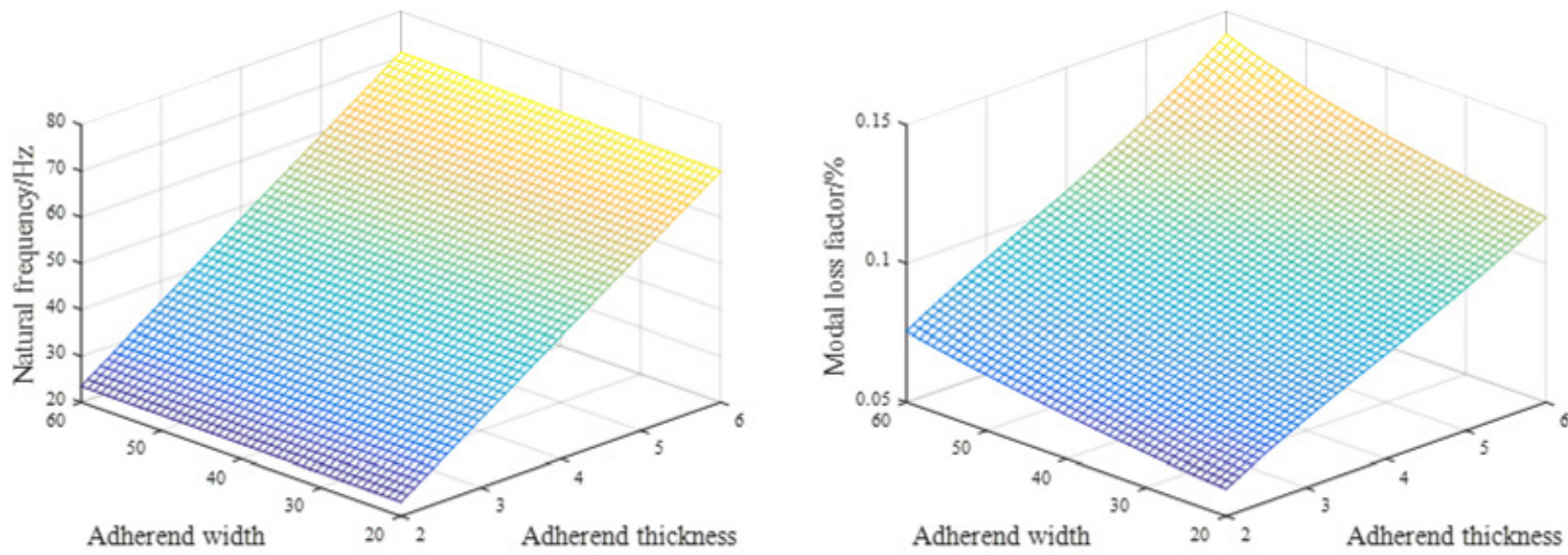

(c) Adherend width-Adherend thickness

Fig. 8 Combined effects of geometrical parameters.

As illustrated in Fig. 8(a), the adherend thickness exhibits considerable effects on natural frequency, since the joint becomes a stiffer structure with increasing adherend thickness. Compared to adherend thickness, the overlap length has 
relatively minor effects on natural frequency. For modal loss factor, both the adherend thickness and overlap length have an impact, but the overlap length is more effective than the adherend thickness. Interesting results are observed in Fig. 8 (b) that the natural frequency exhibits a concave trend with increase of the overlap length. The natural frequency decreases first and then raises at later stage. The resulting surface may be attributed to that more adhesive is used as the overlap length increases. In addition, increasing adherend width would augment the natural frequency and the effect of adherend width on modal loss factor is negligible in comparison with overlap length. The combined effects of adherend thickness and width are shown in Fig. 8(c). It is seen that the adherend width has negligible effects on natural frequency in comparison with adherend thickness. The adherend width and thickness exhibit similar effects on modal loss factor, however, the adherend thickness is more dominant design parameter. In order to explain the concave trend in Fig. 8(b), the variations of the natural frequency versus the adhesive thickness and overlap length are illustrated in Fig. 8(d). The concave trend for thin adhesive layer is not evident, but it becomes apparent when the adhesive layer is thick. The increase of overlap length acts to augment the joint dynamic stiffness, but it will also increase the usage of adhesive, which may reduce the natural frequency. Therefore, balance must be maintained between the two parameters. For thin adhesive layer, the concave trend is not obvious due to that the increase of overlap length will not introduce too much adhesive. However, it is not the case for thick adhesive layer owing to that increasing the overlap length will involve heavy use of adhesive. The natural frequency decreases first until the overlap length dominates the trend. Therefore, blindly increasing the overlap length cannot always increase the natural frequency.

\section{Optimization results}

For optimal design of a general adhesively bonded joint considering vibration characteristics, the objective is often to improve its dynamic stiffness and damping capability. Usually, the improvement of the joint vibration performance will lead to added weight. Therefore, improving the joint free vibration performance with simultaneously controlling its mass is a good subject deserved to investigate. For the adhesively bonded joint, the objective functions can be stated as follows

Minimize: $\phi(\mathbf{x})=\left[\phi_{1}(\mathbf{x}), \phi_{2}(\mathbf{x}), \phi_{3}(\mathbf{x})\right]$

Where

$\phi_{1}=-f_{1}$

$\phi_{2}=-\eta_{1}$

$\phi_{3}=m$

The objective is to maximize the joint dynamic stiffness and damping property, and simultaneously consider minimizing its total weight.

Applying ANNs can reduce the time to calculate natural frequency and loss factor, but a direct search to determine the optimal joint configuration in such a large searching space is still a very time-consuming task. To address the difficulty, genetic algorithm (GA) was implemented to deal with the multi-objective optimization problem. For a multi-objective optimization problem, a set of solutions rather than one optimal solution is obtained. The solutions of multi-objective optimization problem is commonly called Pareto front which represents trade-off between different objectives. In this study, nondominated sorting genetic algorithm (NSGA-II) (Deb et al., 2002), which uses elitism strategy and a crowded comparison operator, was applied to find the Pareto front. The ANNs were used to predict the values of natural frequencies and loss factors for random combinations of design variables.

Based on the framework stated above, an optimization process is conducted to achieve a trade-off among the dynamic bending stiffness (natural frequency of 1 st bending mode, $f_{1}$ ), the damping property (its corresponding modal loss factor, $\eta_{1}$ ) and the total mass. As seen in section 5, the adhesive Poisson's ratio has no significant effects on the joint dynamic behaviors, so its effects were ignored in the whole optimization procedure. Compared to adherend thickness and overlap length, the effects of adherend width are less important on the joint dynamic behaviors and increasing adherend width will increase the joint mass significantly. Though the adherend thickness plays important role 
in the joint vibration properties, the joint added mass is considerably large with increasing adherend thickness. Hence the adhesive Poisson's ratio, adherend width and adherend thickness are kept constant in the whole optimization procedure. The ranges of these design parameters are set as follows: $0.1 \leq \eta_{a} \leq 5, \quad 1 \mathrm{GPa} \leq E_{a} \leq 10 \mathrm{GPa}$, $0.05 \mathrm{~mm} \leq t_{\mathrm{a}} \leq 1.0 \mathrm{~mm}, 30 \mathrm{~mm} \leq L_{\mathrm{o}} \leq 120 \mathrm{~mm}, \quad v_{a}=0.34, h=4 \mathrm{~mm}$ and $w=30 \mathrm{~mm}$. Since the design variables are continuous, the values of which are encoded by real values. The control parameters of NSGA-II in this design case are specified as a population size of 200 , a mutation rate of 0.1 , a crossover rate of 0.9 and an iteration generation of 300 .

The optimization procedure converges after 300 generations and the final Pareto solutions in three dimensions are depicted in Fig. 9(a). Moreover, three different projections of all nondominated solutions are plotted in Fig. 9(b)-(d): (b) plane ( $\left.\phi_{1}, \phi_{2}\right)$, i.e. the maximization of the natural frequency versus the maximization of the modal loss factor; (c) plane $\left(\phi_{1}, \phi_{3}\right)$, i.e. the maximization of the natural frequency versus the minimization of the mass; $(d)$ plane $\left(\phi_{2}, \phi_{3}\right)$, i.e. the maximization of the modal loss factor versus the minimization of the mass. Several representative solutions are marked in these figures and the detailed variable values are listed in Table 2.

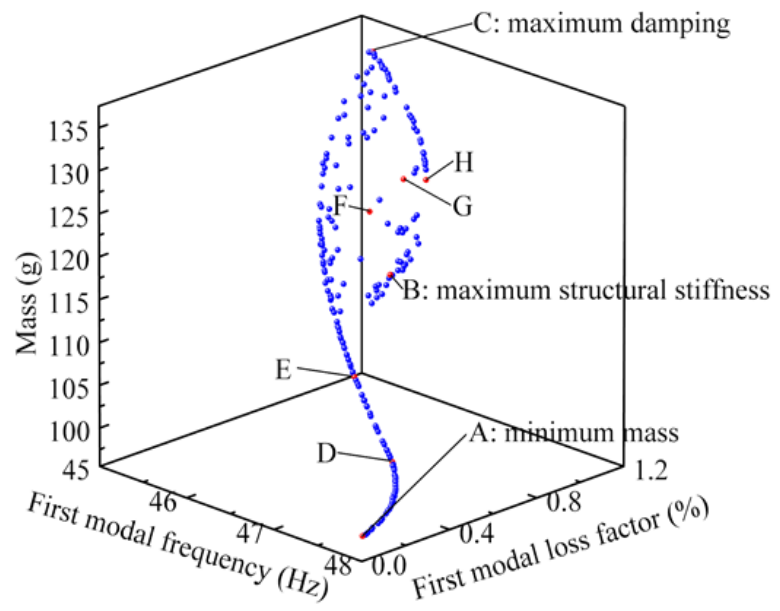

(a)

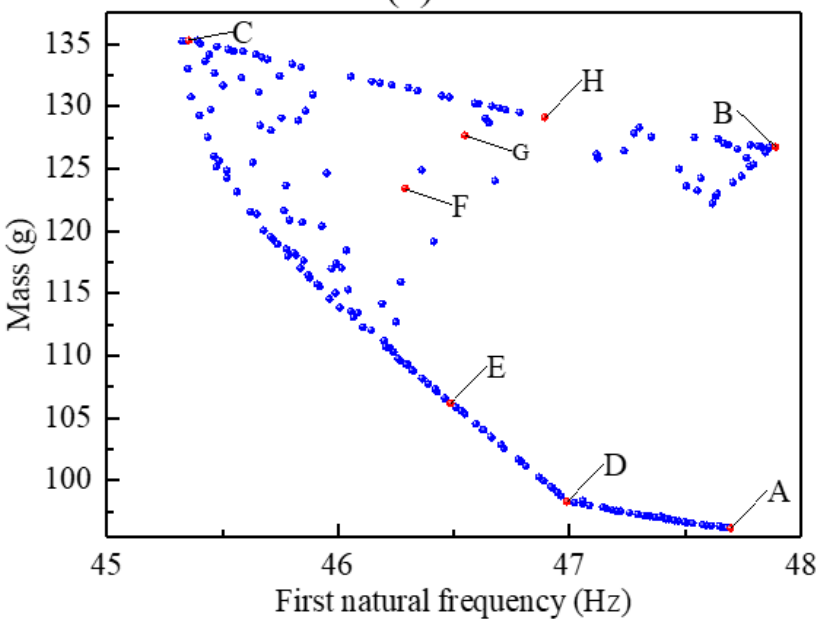

(c)

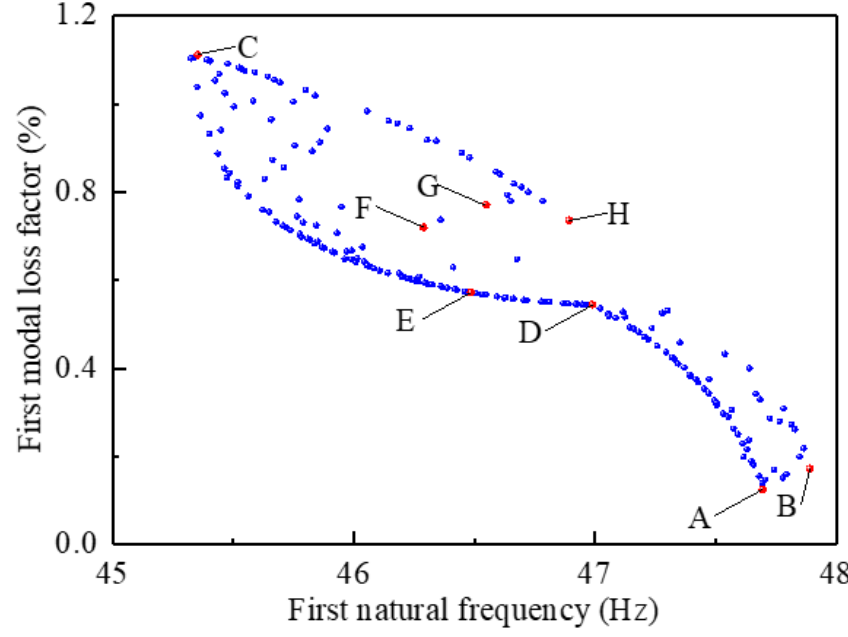

(b)

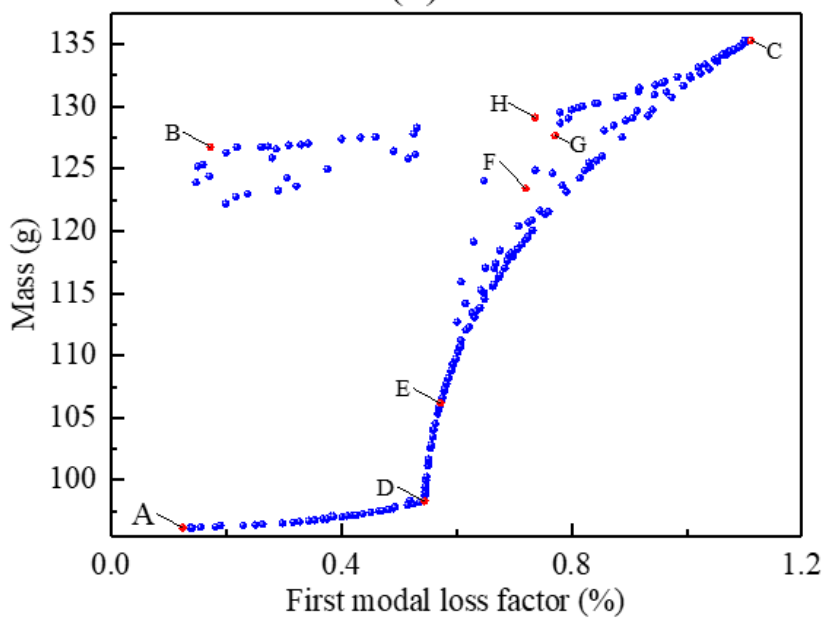

(d)

Fig. 9 Pareto front for the optimization of the adhesive double-strap joint: (a) in 3-dimensional view; (b)-(d) in projection views.

Three extreme solutions are labeled with A, B and C. Solution A gives the optimum design for mass (96.17 g), but the worst for damping property (0.125\%). Moreover, Solution A performs well for dynamic bending stiffness. In this condition, both the adhesive thickness and overlap length take the lower limits of the variable values. Solution $B$ is the optimal solution for bending stiffness $(47.89 \mathrm{~Hz})$, but suffers from high mass (126.75 g) and poor damping property $(0.172 \%)$. In this case, both the adhesive loss factor and overlap length take the upper limits of the variable values, whereas the adhesive thickness reaches the lower limit of the variable value. As mentioned before, the effects of overlap length on natural frequency exhibit a concave trend. For thin adhesive layer, the trend is not evident. Therefore, one can increase overlap length to improve dynamic stiffness for thin adhesive layer. However, the approach will lead to high 
mass and poor damping capability. In addition, it is noted that the adhesive Young's modulus achieves moderate value which simultaneously balances the dynamic stiffness and damping capability. Solution $C$ is the optimal solution for damping, but almost the worst for mass $(135.30 \mathrm{~g})$ and dynamic stiffness $(45.35 \mathrm{~Hz})$. In this circumstance, both the adhesive thickness and overlap length reach the upper limits of the variable values, whereas the adhesive Young's modulus reaches the lower limit of the variable value. It is also noticed that the adhesive loss factor achieves an appropriate value (2.001) to obtain maximum damping capability. These configurations have validated our parametric studies carried out before and proven that NSGA-II can obtain the optimal solutions for the multi-objective optimization problem. Through the analysis, it can be found that the three objectives cannot be achieved at the same time.

Consequently, one can make a trade-off among the three objective functions with aid of the projection plots. As seen in Fig. 9(b), the maximization of dynamic stiffness conflicts with the maximization of damping capability. The Pareto solutions from point $A$ to Point $D$ form a curve in space. These solutions have minimum overlap length $(30 \mathrm{~mm})$. The adhesive thickness increases to improve damping, and thus results in the reduction of the natural frequency. Meanwhile, the increase of adhesive thickness causes sight increase of mass, as shown in Fig. 9(c)-(d). If one wants to achieve a good compromise between the dynamic stiffness and mass, solution $D$ is a good alternative. The Pareto solutions from point $\mathrm{D}$ to $\mathrm{C}$ also form a curve in space and solution $\mathrm{E}$ is in the curve. These solutions have maximum adhesive thickness. The overlap length increases to improve damping, but causes the reduction of natural frequency. As seen in Fig. 8(d), for thick adhesive layer (e.g., $1 \mathrm{~mm}$ ), the natural frequency decreases with increase of overlap length (less than $120 \mathrm{~mm}$ ). Also, the increase of overlap length will increase the mass significantly. If one wants to obtain a good compromise between dynamic stiffness, damping property and mass, solution $\mathrm{E}$ is a good alternative. The Pareto solutions from point $\mathrm{C}$ to point $\mathrm{B}$ form another curve in space and solution $\mathrm{H}$ is in the curve. These solutions have maximum overlap length $(120 \mathrm{~mm})$. The thickness of adhesive layer decreases to improve dynamic stiffness, along with the reduction of damping capability. Meanwhile, these solutions suffer from high mass. If one wants to achieve a good compromise between dynamic stiffness and damping property, solution $\mathrm{H}$ is a good alternative. Besides, it is seen that some candidate solutions such as solution $\mathrm{F}$ and $\mathrm{G}$ scatter between the curves. These solutions have intermediate overlap length and adhesive thickness, which can give comparable damping capability and dynamic stiffness but bring more added mass. Furthermore, it is noted these candidate solutions prefer adhesive loss factor with value near 2 and Young's modulus with $1 \mathrm{GPa}$ (except for solution B aiming to maximize dynamic stiffness). It should also be noticed that the natural frequency varies in a relatively small range for the current considered ranges of design variables. If one wants to achieve larger natural frequency, increasing adherend thickness or overlap length is necessary. Nevertheless, there will be significant increase in structural mass.

From the application, it is obvious that NSGA-II in conjunction with the ANNs have great design flexibility for the adhesively bonded double-strap joint. Multiple optimal objects are handled simultaneously and the required trade-offs between the free vibration behaviors and structural mass can be attained.

Table 2 Pareto-optimal alternatives marked in Fig. 9.

\begin{tabular}{cccccccc}
\hline \multirow{2}{*}{ Solutions } & \multicolumn{4}{c}{ Design variables } & \multicolumn{3}{c}{ Objective functions } \\
\cline { 2 - 7 } & $\eta_{\mathrm{a}}$ & $t_{\mathrm{a}} / \mathrm{mm}$ & $E_{a} / \mathrm{GPa}$ & $L_{\mathrm{o}} / \mathrm{mm}$ & $f_{1} / \mathrm{Hz}$ & $\eta_{1} / \%$ & $\mathrm{~m} / \mathrm{g}$ \\
\hline A & 1.926 & 0.05 & 1.19 & 30.0 & 47.70 & 0.125 & 96.17 \\
B & 5.000 & 0.05 & 1.72 & 120.0 & 47.89 & 0.172 & 126.75 \\
C & 2.001 & 1.00 & 1.00 & 120.0 & 45.35 & 1.111 & 135.30 \\
D & 1.789 & 1.00 & 1.00 & 30.0 & 46.99 & 0.545 & 98.31 \\
E & 1.829 & 1.00 & 1.00 & 49.2 & 46.49 & 0.572 & 106.19 \\
F & 2.072 & 0.56 & 1.00 & 99.0 & 46.29 & 0.720 & 123.43 \\
G & 2.206 & 0.44 & 1.00 & 113.0 & 46.55 & 0.771 & 127.68 \\
H & 2.117 & 0.31 & 1.00 & 120.0 & 46.89 & 0.736 & 129.12 \\
\hline
\end{tabular}

\section{CONCLUSION}

In this paper, the free vibration analysis and optimal design of an adhesive double-strap joint with viscoelastic layer were performed. By introducing virtual boundary conditions, a layerwise element model was extended for modeling the joint. The model was then validated by three-dimensional solid finite element model. ANNs were subsequently established through the training data given by the proposed layerwise finite element model to predict the joint first 
natural frequency and corresponding loss factor. Based on the trained ANNs, parametric studies were carried out to investigate the effects of adhesive material properties and joint geometrical parameters on the joint vibration behaviors. Finally, a multi-objective optimization assisted by NSGA-II was implemented successfully to improve the joint dynamic stiffness and damping while minimizing the total weight.

It is shown that the present numerical results agree with those obtained by three-dimensional finite element model well, except for some discrepancies in loss factor for lateral vibrations. Considering that little attention is given to the joint lateral vibrations, the present layerwise finite element approach is a good tool to analyze the joint free vibration. Besides, the trained ANNs can accurately predict the modal frequency and loss factor on test set. This enables us to conduct parametric studies without enormous numerical calculations. For adhesive layer, increasing its thickness will improve damping, but will decrease dynamic stiffness. Additionally, blindly increasing adhesive loss factor cannot always increase damping and the value of 2 seems appropriate. The adhesive Young's modulus exhibits significant effects on damping when it is small (less than $2 \mathrm{GPa}$ ), but the effects are minor when it is large (more than $6 \mathrm{GPa}$ ). Slight changes are encountered for variations of adhesive Poisson's ratio, so it keeps constant in optimization. For joint geometry parameters, the adherend thickness has considerable effects on dynamic stiffness and the overlap length plays important role in damping capability. The overlap length exhibits concave trend for natural frequency. This becomes more evident when the adhesive layer is thick. Compared to adherend thickness and overlap length, the adherend width has less effects on joint vibration behaviors. Based on NSGA-II, a multi-objective optimization was implemented. The solutions indicate that NSGA-II can successfully find the Pareto front. Through the optimal results, it can be found that the configurations with the adhesive Young's modulus of $1 \mathrm{GPa}$ and loss factor near 2 are preferred.

It is proved that the combination of the present layerwise finite element model, ANNs and NSGA-II provides an efficient tool on the design of adhesively bonded joints. Further multi-objective optimization considering both the static and dynamic behaviors of the joint will be carried out in the future.

Author's Contributions: Conceptualization, Q Guo; Data curation, Q Guo; Formal analysis, Q Guo; Investigation, Q Guo; Methodology, Q Guo; Resources, S Wang; Software, S Wang; Supervision, S Wang; Validation, S Wang; Visualization, S Wang; Writing - original draft, Q Guo; Writing - review \& editing, Q Guo.

Editor: Marco L. Bittencourt.

\section{References}

Almitani KH, Othman R. Analytical solution of the harmonic response of visco-elastic adhesively bonded single-lap and doublelap joints. Int J Adhes Adhes 2016;71:55-65.

Apalak MK, Ekici R, Yildirim M. Optimal design of an adhesively-bonded corner joint with single support based on the free vibration analysis. J Adhes Sci Technol 2006;20:1507-28.

Apalak ZG, Ekici R, Yildirim M, Apalak MK. Free vibration analysis of an adhesively bonded functionally graded double containment cantilever joint. J Adhes Sci Technol 2014;28:1117-39.

Araújo AL, Mota Soares CM, Mota Soares CA, Herskovits J. Optimal design and parameter estimation of frequency dependent viscoelastic laminated sandwich composite plates. Compos Struct 2010;92:2321-7.

Challita G. Analytical study of the dynamic behavior of a voided adhesively bonded lap joint under axial harmonic load. Int J Solids Struct 2018;141-142:183-94.

Deb K, Pratap A, Agarwal S, Meyarivan T. A fast and elitist multiobjective genetic algorithm: NSGA-II. IEEE Trans Evol Comput 2002;6:182-97.

Du Y, Shi L. Effect of vibration fatigue on modal properties of single lap adhesive joints. Int J Adhes Adhes 2014;53:72-9.

Gunes R, Apalak MK, Yildirim M. The free vibration analysis and optimal design of an adhesively bonded functionally graded single lap joint. Int J Mech Sci 2007;49:479-99.

Gunes R, Kemal Apalak M, Yildirim M, Ozkes I. Free vibration analysis of adhesively bonded single lap joints with wide and narrow functionally graded plates. Compos Struct 2010;92:1-17. 
He S, Rao MD. Longitudinal vibration and damping analysis of adhesively bonded double-strap joints. J Vib Acoust 1992a;114:330.

He S, Rao MD. Vibration analysis of adhesively bonded lap joint, part I: Theory. J Sound Vib 1992b;152:405-16.

He X, Oyadiji SO. Influence of adhesive characteristics on the transverse free vibration of single lap-jointed cantilevered beams. J Mater Process Technol 2001;119:366-73.

He X. Finite element analysis of torsional free vibration of adhesively bonded single-lap joints. Int J Adhes Adhes 2014;48:5966.

He X. Numerical and experimental investigations of the dynamic response of bonded beams with a single-lap joint. Int J Adhes Adhes 2012;37:79-85.

Ingole SB, Chatterjee A. Vibration analysis of single lap adhesive joint: experimental and analytical investigation. J Vib Control 2011;17:1547-56.

Jones R M. Mechanics of composite materials. CRC press, 2014.

Kaya A, Tekelioğlu MS, Findik F. Effects of various parameters on dynamic characteristics in adhesively bonded joints. Mater Lett 2004;58:3451-6.

Ko T-C, Lin C-C, Chu R-C. Vibration of bonded laminated lap-joint plates using adhesive interface elements. J Sound Vib 1995;184:567-83.

Lehoucq RB, Sorensen DC. Deflation techniques for an implicitly restarted arnoldi iteration. SIAM J Matrix Anal Appl 1996;17:789-821.

Lin C-C, Ko T-C. Free vibration of bonded plates. Comput Struct 1997;64:441-52.

Liu B, Zhao L, Ferreira AJM, Xing YF, Neves AMA, Wang J. Analysis of viscoelastic sandwich laminates using a unified formulation and a differential quadrature hierarchical finite element method. Compos Part B Eng 2017;110:185-92.

Moreira RAS, Rodrigues JD. A layerwise model for thin soft core sandwich plates. Comput Struct 2006a;84:1256-63.

Moreira RAS, Rodrigues JD, Ferreira AJM. A generalized layerwise finite element for multi-layer damping treatments. Comput Mech 2006b;37:426-44.

Morris MD, Mitchell TJ. Exploratory designs for computational experiments. J Stat Plan Inference 1995;43:381-402.

Paygozar B, Sadigh MAS. Detection of Cracks in Adhesively Bonded Double-Strap Joints Using Artificial Neural Network Method. J Fail Anal Prev 2017;17:1023-30.

Rao MD, Crocker MJ. Analytical and experimental study of the vibration of bonded beams with a lap joint. J Vib Acoust $1990 ; 112: 444$

Rao MD, He S. Analysis of natural frequencies and modal loss factors of simply supported beams with adhesively bonded double-strap joints. J Acoust Soc Am 1992a;92:268-76.

Rao MD, He S. Vibration analysis of adhesively bonded lap joint, part II: Numerical solution. J Sound Vib 1992b;152:417-25.

Rao MD, Zhou H. Vibration and damping of a bonded tubular lap joint. J Sound Vib 1994;178:577-90.

Ren S, Zhao G, Zhang S. A layerwise finite element formulation for vibration and damping analysis of sandwich plate with moderately thick viscoelastic core. Mech Adv Mater Struct 2019;0:1-12.

Saito H, Tani H. Vibrations of bonded beams with a single lap adhesive joint. J Sound Vib 1984;92:299-309.

Vaziri A, Hamidzadeh HR, Nayeb-Hashemi H. Dynamic response of adhesively bonded single-lap joints with a void subjected to harmonic peeling loads. Proc Inst Mech Eng Part K J Multi-Body Dyn 2001;215:199-206.

Vaziri A, Nayeb-Hashemi H. Dynamic response of tubular joints with an annular void subjected to a harmonic torsional load. Proc Inst Mech Eng Part K J Multi-Body Dyn 2002;216:361-70.

Wang S, Li Y, Xie Z. Free vibration analysis of adhesively bonded lap joints through layerwise finite element. Compos Struct 2019;223:110943. 
Xing BY, He XC, Feng MS. Influence of Adhesive Dimensions on the Transverse Free Vibration of Single-Lap Adhesive Cantilevered Beams. Adv Mater Res 2011;393-395:149-52.

Xu C, Lin S, Yang Y. Optimal design of viscoelastic damping structures using layerwise finite element analysis and multiobjective genetic algorithm. Comput Struct 2015;157:1-8.

Zapfe JA, Lesieutre GA. A discrete layer beam finite element for the dynamic analysis of composite sandwich beams with integral damping layers. Comput Struct 1999;70:647-66. 